\title{
Clinical value of CTLA4 combined with clinicopathological factors in evaluating the prognosis of breast cancer
}

\author{
Junyi $\mathrm{Wu}^{1 \#}$, Lei $\mathrm{Li}^{2 \#}$, Jiayi Chen ${ }^{2}$, Yuan Liu ${ }^{2}$, Junming Xu ${ }^{2}$, Zhihai Peng ${ }^{1}$ \\ ${ }^{1}$ Department of General Surgery, Shanghai General Hospital of Nanjing Medical University, Shanghai, China; ${ }^{2}$ Department of General Surgery, \\ Shanghai General Hospital, Shanghai Jiao Tong University School of Medicine, Shanghai, China \\ Contributions: (I) Conception and design: J Wu, Z Peng; (II) Administrative support: Z Peng; (III) Provision of study materials or patients: L Li, J \\ Chen; (IV) Collection and assembly of data: J Wu, L Li; (V) Data analysis and interpretation: Y Liu, J Xu; (VI) Manuscript writing: All authors; (VII) \\ Final approval of manuscript: All authors. \\ "These authors contributed equally to this work. \\ Correspondence to: Zhihai Peng. Shanghai General Hospital of Nanjing Medical University, 100 Haining Road, Hongkou, Shanghai 200080, China. \\ Email: pengzhihai1958@163.com.
}

Background: Clinical prediction of breast cancer prognosis relies on both clinical-pathological features and biological markers. Many studies have revealed that tumor cytotoxic T lymphocyte antigen 4 (CTLA4) expression may present prognostic predicting value in cancers. We intended to explore the prognostic value of significant clinicopathological parameters and CTLA4 for predicting survival of patients with breast cancer.

Methods: A total of 229 breast cancer patients who had radical surgery treatment between Sep 2009 and April 2011 were enrolled in this study. Immunohistochemical staining was performed to evaluate CTLA4 grade and Ki-67 index in breast cancer tissue. Univariate and multivariate logistic analysis, Kaplan-Meier survival analysis and ROC curve were used to explore the association between CTLA4 or clinicopathological parameters and disease-free survival (DFS). A nomogram was constructed based on the regression model to predict DFS of patients with breast cancer.

Results: CTLA4 grade (OR 1.730, 95\% CI: 1.213-2.468, P=0.002), Ki-67 (OR 1.449, 95\% CI: 1.0691.964, $\mathrm{P}=0.017$ ) and $\mathrm{N}$ stage (lymph node metastasis) (OR 2.268, 95\% CI: 1.588-3.303, P=0.000) showed significantly association with DFS of breast cancer patients. All these factors were independent predictors for poor survival, as patients with stage N2-3 tumors, high CTLA4 grade and Ki-67 index showed low survival probability $(\mathrm{P}<0.01)$. The conjunction of these factors exhibited good discrimination value (AUC 0.815 , 95\% CI: 0.749-0.882, P=0.000). Nomogram performed based on CTLA4 grade, Ki-67 index and N stage provided an efficient method to predict DFS of patients with breast cancer.

Conclusions: The high expression of CTLA4 and Ki-67 together with lymph node metastasis in breast cancer are independent risk factors that affect the prognosis of breast cancer patients. They have the potentiality to be utilized conjunctively as predictor in clinical practice.

Keywords: Breast cancer; nomogram; cytotoxic T lymphocyte antigen 4 (CTLA4); Ki-67 index; N stage

Submitted Mar 17, 2020. Accepted for publication Aug 16, 2020.

doi: $10.21037 /$ gs-20-359

View this article at: http://dx.doi.org/10.21037/gs-20-359

\section{Introduction}

Current clinical prediction of breast cancer prognosis relies on both clinical-pathological features and biological markers
(1). Estrogen receptor (ER), progesterone receptor (PR), epidermal growth factor receptor 2 (HER2) and Ki-67 have been universally applied to divide breast cancer into subtypes, as well as used as predictive factors for evaluating 
prognosis in clinical practice. As adjuvant endocrine therapy shows high effectiveness in treating ER/PR positive breast cancer, these subtypes are supposed to have better prognosis than triple negative breast cancer (TNBC) $(2,3)$. HER2 overexpression in the absence of anti-HER-2 targeted therapy is associated with adverse prognosis (3). Ki-67, indicating proliferation index, has been widely accepted as a good predictive and prognostic marker in clinical practice (2).

Recently, as increasing knowledge in the field of interplay between immune system and cancer, immunotherapies are gaining its popularity in treatment of a large range of cancers $(4,5)$. Cytotoxic $\mathrm{T}$ lymphocyte antigen 4 (CTLA4) is a molecule that plays an inhibitory role in the proliferation and activation of $\mathrm{T}$ cells. It can competitively combine with costimulatory molecules B7-1 and B7-2 (also known as CD80 and CD86) expressed on antigenpresenting cells to transmit costimulatory signals to $\mathrm{T}$ cells (4-6). Thus, CTLA4 takes part in the processes of maintaining the homeostasis and self-tolerance of immune system under physiological condition. In diseases related to immune dysfunction, CTLA4 blockade is supposed to relieve the immune response of $\mathrm{T}$ cell and has been applied as one of the immune checkpoints inhibitors in several malignancies especially melanoma and none small cell lung cancer (NSCLC) (7-9).

In breast cancer, immune-related factors contribute to ascertain patients' response to immunotherapy as well as prognosis, such as tumor-infiltrating lymphocytes and immune-related gene signatures (1). Previous studies have revealed that tumor CTLA-4 expression may present prognostic predicting value in breast cancer, but no consensus has been reached $(10,11)$. Although the increasingly evidences reveals that immune-related factors are associated with the prognosis of cancer, the clinicalpathological features of cancer still hold an unsubstitutable position in forecasting outcome of patients. Our study aimed to further explore the value of significant clinicalpathological features and CTLA4 in predicting the survival of patients suffering from breast cancer. We present the following article/case in accordance with the STROBE reporting checklist (available at http://dx. doi. org/10. 21037/gs-20-359).

\section{Methods}

\section{Patients}

In this study, we selected a total of 229 cases of breast cancer patients who underwent surgical treatment between Sep 2009 and April 2011 at the Shanghai general hospital. We included patients who were pathologically diagnosed with breast cancer and willing to participate in follow-up. We excluded patients who received preoperative radiotherapy and chemotherapy or had recurrent breast cancer. The study was approved by the Ethics Committee of Shanghai General Hospital of Nanjing Medical University (No. 2020SQ137) and was conducted in accordance with the Declaration of Helsinki (as revised in 2013). As this study is a retrospective study and contents of the study do not involve personal privacy, the ethics committee approves this study is exempt from the informed consent of participants.

\section{Data collection}

The clinical-pathological characteristics of 229 patients were gathered by well-trained research fellows. The patients' characteristics included age at initial diagnosis and menstrual status. The tumor characteristics concentrated on pathological type, histological grade, tumor size, lymph node metastasis, Ki-67 index, CTLA4 grade and molecular type (10-12).

\section{Immunobistochemistry}

Immunohistochemistry was applied to stain $\mathrm{Ki}-67$ and CTLA4 in paraffin sections of representative breast cancer tissues. Murine anti-CTLA-4 and anti-Ki-67 mAbs were used for immunohistochemical staining and 3,3'-diaminobenzidine (DAB) Detection Kit was used for color developing. CTLA4 grade was recorded as 0 (negative), 1 (weak), 2 (intermediate), 3 (strong) according to the percentage and intensity of CTLA-4 in tumors $(10,13)$. The scores of percentage of positive tumor cells were recorded as: 0 (negative), 1 (1-30\%), 2 (31-60\%) or 3 (61-100\%). The scores of intensity of positive tumor cells were recorded as: 0 (negative), 1 (weak), 2 (moderate) or 3 (strong). The final grade was recorded as $0(0$, negative), I (2-3, weak), II (4-6, intermediate), III (>6, strong) (Figure 1) (14). The Ki-67 index was assessed as a percentage of stained nuclei, and were classified as low ( $<20 \%$ immunoreactivity) or high $(\geq 20 \%$ immunoreactivity) based on the percentage of Ki-67-positive cells.

\section{Statistical analysis}

Quantitative variables were expressed as the mean \pm 


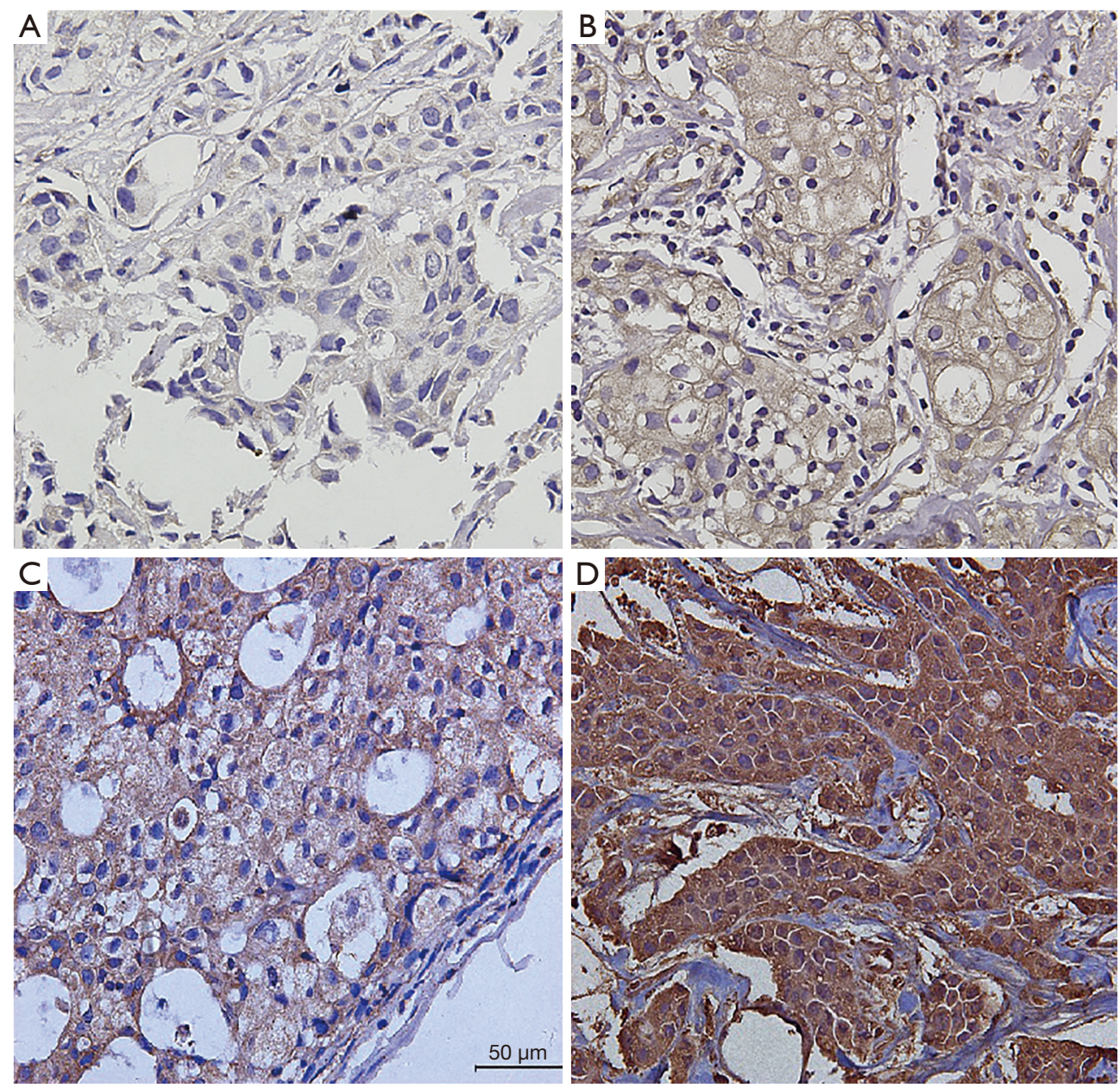

Figure 1 Immunohistochemical staining of CTLA4 in paraffin sections of breast cancer tissue. Immunohistochemical staining of CTLA4 in paraffin sections of representative breast cancer tissue shows four grades. Murine anti-CTLA-4 mAb was used for immunohistochemical staining and DAB Detection Kit was used for color developing. CTLA4 grade was recorded as 0 (negative) (A), I (weak) (B), II (intermediate) (C), III (strong) (D), magnification $\times 400$.

standard deviation (SD) and compared by Student's $t$-test. Categorical variables were presented as values (percentages) and compared using Fisher's exact test or Pearson's $\chi^{2}$ test. Univariate and multivariate analysis were performed using logistic regression model to evaluate the prognostic value of involved variables. Odds ratio (OR) and $95 \%$ confidence index (95\% CI) were calculated. Kaplan-Meier method were applied to estimate survival probabilities stratified by significant factors. Receiver-operating characteristic (ROC) curve was used to determine the sensitivity and specificity of prognostic factors. Nomogram was constructed based on the logistic regression analysis to integrate different factors to predict the survival of breast cancer patients.

SPSS version 22.0 (SPSS Inc., Chicago, IL, USA) and
GraphPad PRISM 7 (GraphPad Prism Software Inc., San Diego, California) softwares were used to accomplish the above statistical analyses. A two-sided $\mathrm{P}<0.05$ was deemed to be statistically significant.

\section{Results}

\section{Baseline characteristics}

Of the 229 breast cancer patients, the 1, 2 and 5 -year disease-free survival rates were $98.7 \%, 93.9 \%$ and $77.1 \%$. The baseline clinico-pathological features of the patients are shown in Table 1. The median age at initial diagnosis was 52.3 (range, 25-79). A total of 194 patients (84.7\%) 

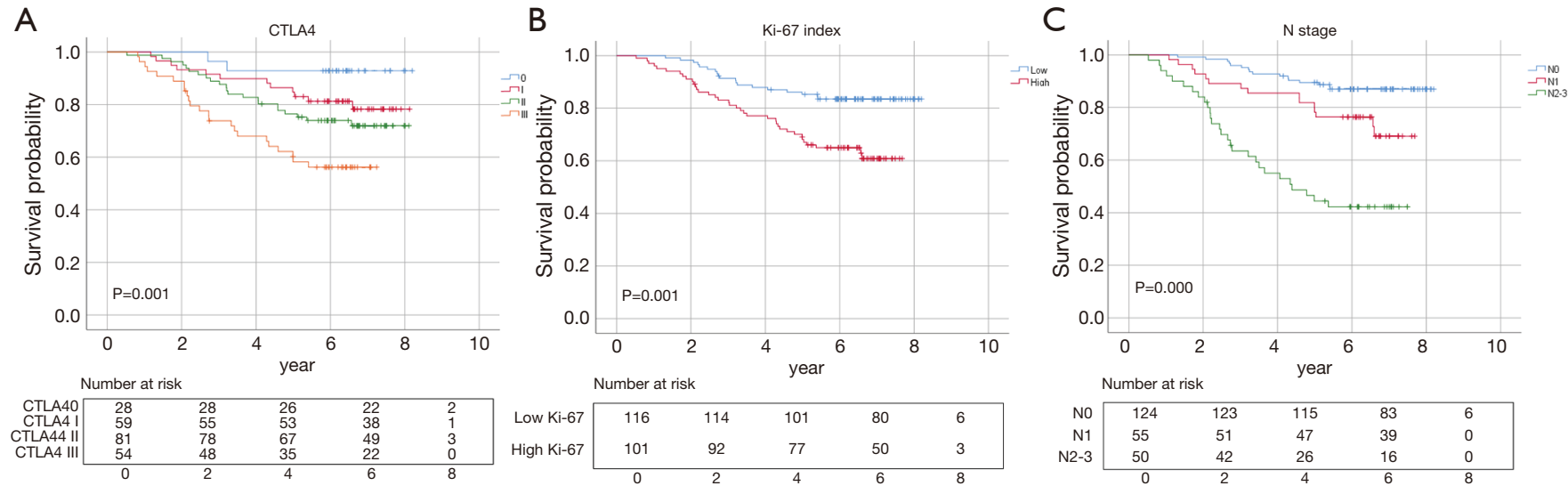

Figure 2 Survival curve about CTLA4 grade, Ki-67 index, and N stage. Kaplan-Meier survival curves showing the correlation between CTLA4 grade/Ki-67 index/N stage and prognosis. (A) Disease-free Survival probability of CTLA4 grade (P=0.001). (B) Disease-free Survival probability of Ki-67 index $(\mathrm{P}=0.0001)$. (C) Disease-free Survival probability of N stage $(\mathrm{P}=0.000)$.

were diagnosed with invasive ductal carcinoma and pathological grade I, II, III tumors were documented in $4.6 \%, 60.3 \%$ and $35.2 \%$ of the patients; 85 patients (38.1\%) were classified with $\mathrm{T} 1$ tumors and others were with $\mathrm{T} 2-3$ tumors; 124 patients $(54.1 \%)$ showed negative lymph node metastasis, and others showed N1 status $(24.0 \%)$ or $\mathrm{N} 2-3$ status $(21.8 \%)$. There were 28 patients $(12.6 \%)$ in the CTLA4-0 group, 59 patients (26.6\%) in the CTLA4-I group, 81 patients $(36.5 \%)$ in the CTLA4-II group, and 54 patients $(24.3 \%)$ in the CTLA4-III group. The distribution of CTLA4 varied significantly according to different tumor histological grade, T stage, $\mathrm{N}$ stage, Ki-67 index, and molecular subtypes (all $\mathrm{P}<0.05$, Table 2).

\section{Univariate and multivariate analysis}

By univariate analysis and multivariate analysis, CTLA4 grade, Ki-67 index, $\mathrm{N}$ stage and molecular type was found to be correlated to recurrence of breast cancer. Multivariate analysis by Cox regression showed that CTLA4 grade, Ki67 index and $\mathrm{N}$ stage were independent prognostic factors. High CTLA4 grade was associated with poor disease-free survival of breast cancer (OR 1.730, 95\% CI: 1.213-2.468, $\mathrm{P}=0.002$ ), so was high Ki-67 index (OR 1.449, 95\% CI: 1.069-1.964, $\mathrm{P}=0.017$ ) and $\mathrm{N}$ stage (OR 2.268, 95\% CI: 1.588-3.303, $\mathrm{P}=0.000$ ) (Table 1, Figure 2).

In luminal breast cancer, the recurrence rate of patients increased and the disease-free survival obviously decreased, with the present of these risk factors (CTLA4: $\chi^{2}=12.092$, $\mathrm{P}=0.007$; Ki-67 index: $\chi^{2}=17.990, \mathrm{P}=0.000 ; \mathrm{N}$ stage: $\left.\chi^{2}=39.655, \mathrm{P}=0.000\right)$. However, it was only observed, in triple-negative breast cancer, that the disease-free survival of patients with stage N2-3 tumors was significantly lower than those without lymph node metastasis (N2-3 vs. N0: $\chi^{2}=4.257, P=0.039$ ), and that the disease-free survival of patients with CTLA4 III tumors was lower than those with CTLA4 II and I tumors (CTLA4 III vs. CTLA4 I: $\chi^{2}=4.084$, $\mathrm{P}=0.043$; CTLA4 III $v s$. CTLA4 II: $\chi^{2}=3.590, \mathrm{P}=0.058$ ).

\section{ROC curve and nomogram}

The efficiency of CTLA4 grade, Ki-67 index together with $\mathrm{N}$ stage on predicting the prognostic was assessed by ROC curve. As the result demonstrated, they showed good predictive value for disease-free survival of patients [area under curve (AUC) 0.815, 95\% CI: 0.749-0.882, $\mathrm{P}=0.000$, Figure 3). Further, nomogram was established on the basis of these three factors to predict survival (Figure 4). By adding up each score of the variables and getting a total point, a corresponding predicted value of survival was obtained at the bottom of the figure.

\section{Discussion}

Immunotherapies are gaining its popularity for treatment of a large range of cancers and one of the well-known immunotherapy is immune checkpoint blockade, of which the best two are blockades targeting CTLA4 and programmed death-1 (PD-1). They can relieve inhibitory signals of T-cell activation and thus enhance effective 
Table 1 Characteristics of 229 eligible patients

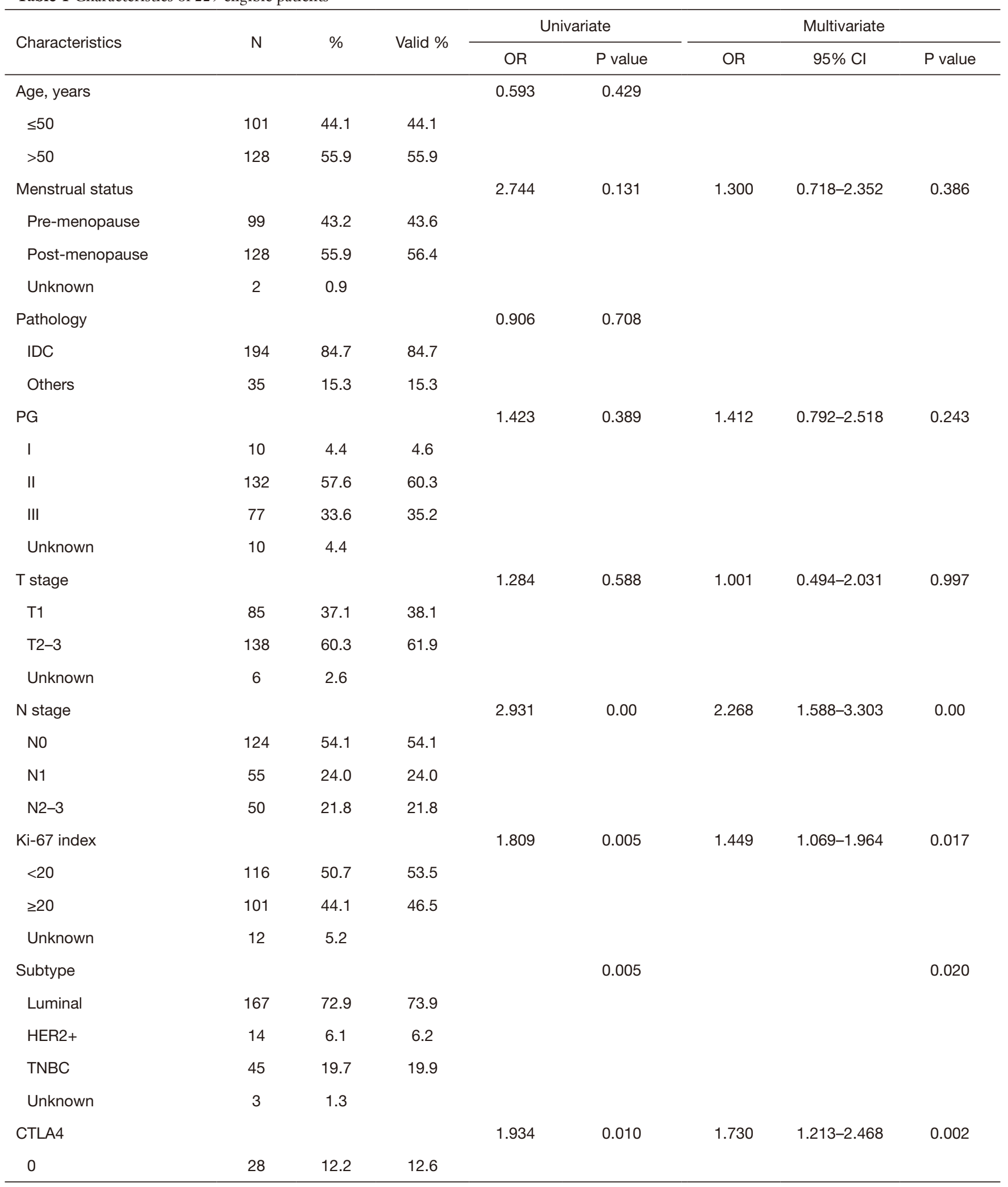

Table 1 (continued) 
Table 1 (continued)

\begin{tabular}{|c|c|c|c|c|c|c|c|c|}
\hline Characteristics & $\mathrm{N}$ & $\%$ & Valid \% & \multicolumn{2}{|c|}{ Univariate } & \multicolumn{3}{|c|}{ Multivariate } \\
\hline I & 59 & 25.8 & 26.6 & & & & & \\
\hline II & 81 & 35.4 & 36.5 & & & & & \\
\hline III & 54 & 23.6 & 24.3 & & & & & \\
\hline Chemotherapy & & & & 0.344 & 0.104 & 0.587 & $0.231-1.494$ & 0.264 \\
\hline No chemotherapy & 23 & 10.0 & 10.1 & & & & & \\
\hline Chemotherapy & 205 & 89.5 & 89.9 & & & & & \\
\hline Unknown & 1 & 0.4 & & & & & & \\
\hline
\end{tabular}

PG, pathological grade; HER2, human epidermal growth receptor 2; TNBC, triple-negative breast cancer; CTLA4, cytotoxic T lymphocyte antigen 4; OR, odds ratio; $\mathrm{Cl}$, confidence interval.

antitumor response (4-6). Anti-CTLA4 and anti-PD1 have been verified to be safe and active in treating advanced melanoma and NSCLC in clinical trials, bringing favorable survival improvement (14-16).

It has been reported recently that CTLA4 is also expressed in various solid tumors apart from $\mathrm{T}$ cell (17-20). Theoretically, the CTLA4 expressed by tumor cells is supposed to facilitate tumor cell evasion through ablating the immune surveillance of immune cells around the tumor (21). However, the clinical value of CTLA4 in predicting prognosis of various cancer still remains ambiguous. Tumor CTLA4 overexpression was associated with shorter overall survival (OS) and could be an independent prognostic predictor in esophageal carcinoma and laryngeal and pharyngeal squamous cell carcinoma $(17,18)$. While a study investigating CTLA4 expression and prognostic role in 81 patients with radically resected stage I-III NSCLC came to a contrary conclusion that CTLA4 overexpression had a positive effect on OS (19). Besides, another study showed that there is no significant difference occurring in the OS of gastric cancer patients with low or high CTLA4 expression (20).

In breast cancer, previous study has uncovered that CTLA4 polymorphisms significantly associate with breast cancer susceptibility in Asian populations (22). The mRNA expression of CTLA4 in unstimulated PBMCs from patients with breast cancer were significantly higher than healthy control (23). Anti-CTLA4 therapy has been proved to be effective in clinical treatment of breast cancer $(24,25)$. In luminal B HER2-negative breast cancer, tumor
$\mathrm{CTLA}^{+}$patients had shorter disease-free survival (DFS), so did the interstitial CTLA4 $4^{+}$patients. Tumor CTLA4+ was proved an independent predictor of shorter DFS (11). In this study, we used immunohistochemistry to analyze the levels of CTLA4 in breast cancer tissues and then classified the patients into four grades. Here, we showed that the distribution of CTLA4 varied significantly according to different tumor histological grade, $\mathrm{T}$ stage, $\mathrm{N}$ stage, Ki67 index and molecular subtypes. In addition, CTLA4 grade also associated with recurrence of breast cancer and confirmed to be an independent prognostic factor. So, the present research adds new opinions in this field and shows innovation and clinical significances.

$\mathrm{Ki}-67$, a nuclear protein, is considered as an objective marker of proliferative activity in a variety of tumors. Ki67 has been shown to have prognostic value in breast cancer, and its predictive efficacy has been proved in several studies. High Ki-67 index $(\geq 20 \%)$ indicates a high recurrence risk. Lymph node metastasis is risk factor vital important in American Joint Committee on Cancer staging system for breast cancer (26). It counts for much in the prognosis of patients with breast cancer indicated by most studies (27-29), which is consistent with our results that number of lymph metastasis ( $\mathrm{N}$ stage) was associated with the survival of patients. The reasons might be that more numbers of lymph metastasis meant less complete resection probability, leading to poor prognosis. In the present study, we evaluated the survival of breast cancer patients based on clinical-pathological variables and CTLA4 grade. We found that CTLA4 grade, Ki-67 index 
Table 2 Distribution of CTLA4 according to clinico-pathologic factors

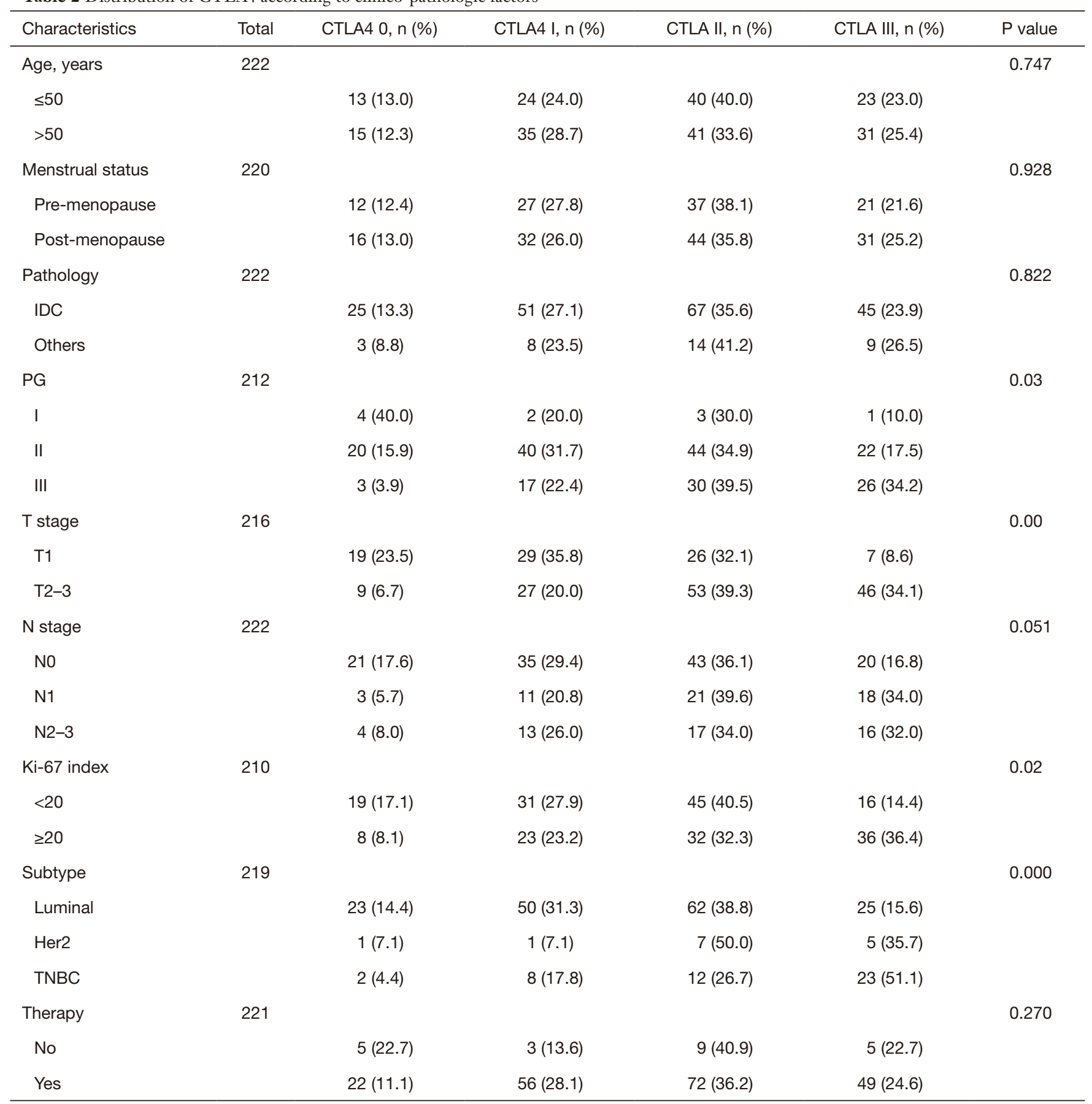

and $\mathrm{N}$ stage was related to poor prognosis of breast cancer patients through multivariate analysis. As CTLA4 grade, $\mathrm{Ki}-67$ index and $\mathrm{N}$ stage increased, the survival probability decreased correspondingly. We further revealed that these independent risk factors could be applied to predict the survival of breast cancer patients effectively.
Nomogram is able to combine various meaningful prognostic variables to predict a specific endpoint and has been constructed to predict survival of various malignancies, including colon cancer (30), prostate cancer (31), clear cell renal cell carcinoma (32) and breast cancer $(33,34)$. While most of the studies combined clinical-pathological variables 
as individual predictors. The present study showed for the first time that CTLA4 together with clinical-pathological variables including $\mathrm{Ki}-67$ index and $\mathrm{N}$ stage functioned

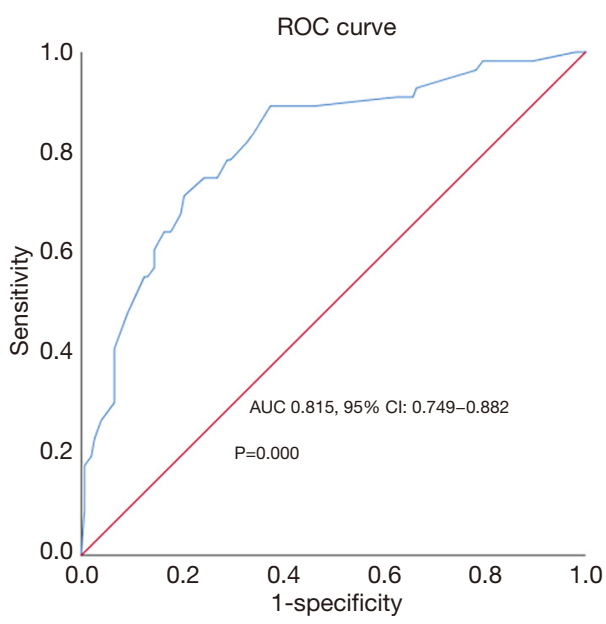

Figure 3 ROC curve of CTLA4, Ki-67 index and N stage. ROC curve was performed to access the prognostic factor: CTLA4 grade, Ki-67 index combined with $\mathrm{N}$ stage (AUC 0.815, 95\% CI: $0.749-0.882, \mathrm{P}=0.000)$. conjunctively to predict the survival of patients with breast cancer. From the known value of the three factors, the clinicians could obtain the predicted survival of individual patients. Hence, this will have important instructional significance for supplementary therapy and follow-up.

\section{Limitations}

When self-reflecting the entire study, we had to admit that the number of patients enrolled in the study was limited, and a larger sample volume would increase the credibility of the study. Besides, our study was performed on the base of a retrospective cohort, which is considered relatively low level of clinical evidence. Prospective clinical trials are required to further verify the validity of CTLA4 grade as the predictors. Considering the controversial conclusion shown in HER2-negative breast cancer, studies directing at specific subtypes of breast cancer are desired for further exploration.

\section{Conclusions}

In conclusion, the present study revealed that CTLA4

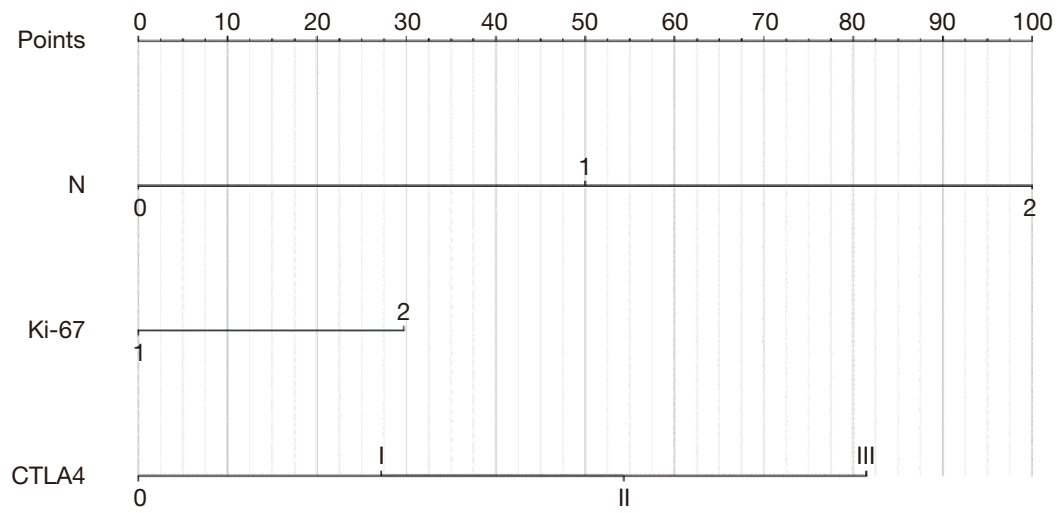

Total points

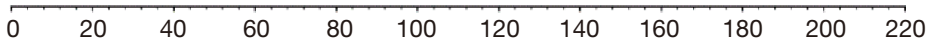

5-year survival probability

$\begin{array}{llllllll}0.95 & 0.9 & 0.8 & 0.7 & 0.6 & 0.5 & 0.4 & 0.3\end{array}$

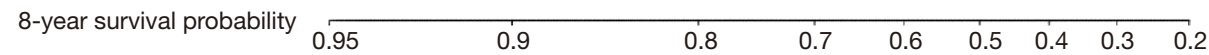

Figure 4 Nomogram according to CTLA4, Ki-67 index and N stage. Nomogram was performed to predict the disease-free survival of patients with breast cancer. 
grade, Ki-67 index and $\mathrm{N}$ stage were reliable and independent factors for predicting the survival of breast cancer. Nomogram based on the three variables may provide clinicians with an effective and convenient method for clinical survival prediction of patients with breast cancer.

\section{Acknowledgments}

Funding: The study was supported by Scientific Research Project of Shanghai Science and Technology Commission (15411962700).

\section{Footnote}

Reporting Checklist: The authors have completed the STROBE reporting checklist. Available at http://dx. doi. org/10.21037/gs-20-359

Data Sharing Statement: Available at http://dx. doi. org/10.21037/gs-20-359

Conflicts of Interest: All authors have completed the ICMJE uniform disclosure form (available at http://dx. doi. org/10.21037/gs-20-359). The authors have no conflicts of interest to declare.

Ethical Statement: The authors are accountable for all aspects of the work in ensuring that questions related to the accuracy or integrity of any part of the work are appropriately investigated and resolved. The study was approved by the Ethics Committee of Shanghai General Hospital of Nanjing Medical University (No. 2020SQ137) and was conducted in accordance with the Declaration of Helsinki (as revised in 2013). As this study is a retrospective study and contents of the study do not involve personal privacy, the ethics committee approves this study is exempt from the informed consent of participants.

Open Access Statement: This is an Open Access article distributed in accordance with the Creative Commons Attribution-NonCommercial-NoDerivs 4.0 International License (CC BY-NC-ND 4.0), which permits the noncommercial replication and distribution of the article with the strict proviso that no changes or edits are made and the original work is properly cited (including links to both the formal publication through the relevant DOI and the license). See: https://creativecommons.org/licenses/by-nc$\mathrm{nd} / 4.0 /$.

\section{References}

1. Criscitiello C, Curigliano G. Immunotherapy of Breast Cancer. Prog Tumor Res 2015;42:30-43.

2. Mosly D, Turnbull A, Sims A, et al. Predictive markers of endocrine response in breast cancer. World J Exp Med 2018;8:1-7.

3. Ross JS, Slodkowska EA, Symmans WF, et al. The HER2 receptor and breast cancer: ten years of targeted antiHER-2 therapy and personalized medicine. Oncologist 2009;14:320-68.

4. Azoury SC, Straughan DM, Shukla V. Immune Checkpoint Inhibitors for Cancer Therapy: Clinical Efficacy and Safety. Curr Cancer Drug Targets 2015;15:452-62.

5. Ribas A, Wolchok JD. Cancer immunotherapy using checkpoint blockade. Science 2018;359:1350-1355.

6. Wei SC, Duffy CR. Fundamental Mechanisms of Immune Checkpoint Blockade Therapy. Cancer Discov 2018;8:1069-86.

7. Retseck J, Nasr A, Lin Y, et al. Long term impact of CTLA4 blockade immunotherapy on regulatory and effector immuneresponses in patients with melanoma. J Transl Med 2018;16:184.

8. Whiteside TL, Demaria S, Rodriguez-Ruiz ME, et al. Emerging Opportunities and Challenges in Cancer Immunotherapy. Clin Cancer Res 2016;22:1845-55.

9. Yang J, Chen J, Wei J, et al. Immune checkpoint blockade as a potential therapeutic target in non-small cell lung cancer. Expert Opin Biol Ther 2016;16:1209-23.

10. Yu H, Yang J, Jiao S, et al. Cytotoxic T lymphocyte antigen 4 expression in human breast cancer: implications for prognosis. Cancer Immunol Immunother 2015;64:853-60.

11. Lan G, Li J, Wen Q, et al. Cytotoxic T lymphocyte associated antigen 4 expression predicts poor prognosis in luminal B HER2-negative breast cancer. Oncol Lett 2018;15:5093-7.

12. Ehinger A, Malmström P, Bendahl PO, et al. Histological grade provides significant prognostic information in addition to breast cancer subtypes defined according to $\mathrm{St}$ Gallen 2013. Acta Oncol 2017;56:68-74.

13. Roncella S, Laurent S, Fontana V, et al. CTLA-4 in mesothelioma patients: tissue expression, body fluid levels and possible relevance as a prognostic factor. Cancer Immunol Immunother 2016;65:909-17.

14. Dobry AS, Zogg CK, Hodi FS. Management of metastatic melanoma: improved survival in a national cohort following the approvals of checkpoint blockade 
immunotherapies and targeted therapies. Cancer Immunol Immunother 2018;67:1833-44.

15. Albertini MR. The age of enlightenment in melanoma immunotherapy. J Immunother Cancer 2018;6:80.

16. Ilie M, Benzaquen J, Hofman V, et al. Immunotherapy in Non-Small Cell Lung Cancer: Biological Principles and Future Opportunities. Curr Mol Med 2017;17:527-40.

17. Hellmann MD, Nathanson T, Rizvi H, et al. Genomic Features of Response to Combination Immunotherapy in Patients with Advanced Non-Small-Cell Lung Cancer. Cancer Cell 2018;33:843-52.e4.

18. Karpathiou G, Casteillo F, Giroult JB, et al. Prognostic impact of immune microenvironment in laryngeal and pharyngeal squamous cell carcinoma: Immune cell subtypes, immuno-suppressive pathways and clinicopathologic characteristics. Oncotarget 2017;8:19310-22.

19. Zhang XF, Pan K, Weng DS, et al. Cytotoxic T lymphocyte antigen-4 expression in esophageal carcinoma: implications for prognosis. Oncotarget 2016;7:26670-9.

20. Chang H, Jung WY, Kang Y, et al. Programmed deathligand 1 expression in gastric adenocarcinoma is a poor prognostic factor in a high CD8+ tumor infiltrating lymphocytes group. Oncotarget 2016;7:80426-34.

21. Bindea G, Mlecnik B, Fridman WH, et al. The prognostic impact of anti-cancer immune response: a novel classification of cancer patients. Semin Immunopathol 2011;33:335-40.

22. Dai Z, Tian T, Wang M, et al. CTLA-4 polymorphisms associate with breast cancer susceptibility in Asians: a meta-analysis. Peerj 2017;5:e2815.

23. Khalife E, Khodadadi A, Talaeizadeh A, et al. Overexpression of Regulatory T Cell-Related Markers (FOXP3, CTLA-4 and GITR) by Peripheral Blood Mononuclear Cells from Patients with Breast Cancer. Asian Pac J Cancer Prev 2018;19:3019-25.

24. Müller P, Kreuzaler M, Khan T, et al. Trastuzumab emtansine (T-DM1) renders HER2 + breast cancer highly susceptible to CTLA-4/PD-1 blockade. Science

Cite this article as: $\mathrm{Wu} \mathrm{J}, \mathrm{Li} \mathrm{L}$, Chen J, Liu Y, Xu J, Peng Z. Clinical value of CTLA4 combined with clinicopathological factors in evaluating the prognosis of breast cancer. Gland Surg 2020;9(5):1328-1337. doi: 10.21037/gs-20-359
Translational Medicine 2015;7:315ra188.

25. Liu L, Wang Y, Miao L, et al. Combination Immunotherapy of MUC1 mRNA Nano-vaccine and CTLA-4 Blockade Effectively Inhibits Growth of Triple Negative Breast Cancer. Mol Ther 2018;26:45-55.

26. Singletary SE, Allred C, Ashley P, et al. Revision of the American Joint Committee on Cancer staging system for breast cancer. J Clin Oncol 2002;20:3628-36.

27. Fu J, Wu L, Jiang M, et al. Clinical Nomogram for Predicting Survival Outcomes in Early Mucinous Breast Cancer. PLoS One 2016;11:e0164921.

28. Lin $\mathrm{Y}, \mathrm{Fu} F$, Lin S, et al. A nomogram prediction for the survival of patients with triple negative breast cancer. Oncotarget 2018;9:32108-18.

29. Witteveen A, Vliegen IM, Sonke GS, et al. Personalisation of breast cancer follow-up: a time-dependent prognostic nomogram for the estimation of annual risk of locoregional recurrence in early breast cancer patients. Breast Cancer Res Treat 2015;152:627-36.

30. Weiser MR, Gönen M, Chou JF, et al. Predicting survival after curative colectomy for cancer: individualizing colon cancer staging. J Clin Oncol 2011;29:4796-802.

31. Smaletz O, Scher HI, Small EJ, et al. Nomogram for overall survival of patients with progressive metastatic prostate cancer after castration.J Clin Oncol 2002;20:3972-82.

32. Zhang G, Wu Y, Zhang J, et al. Nomograms for predicting long-term overall survival and disease-specific survival of patients with clear cell renal cell carcinoma. Onco Targets Ther 2018;11:5535-44.

33. Dai D, Jin H, Wang X. Nomogram for predicting survival in triple-negative breast cancer patients with histology of infiltrating duct carcinoma: a population-based study. Am J Cancer Res 2018;8:1576-85.

34. Sun W, Jiang YZ, Liu YR, et al. Nomograms to estimate long-term overall survival and breast cancer-specific survival of patients with luminal breast cancer. Oncotarget 2016;7:20496-506. 